Pacific Journal of Mathematics

ON TWO POLYNOMIAL SPACES ASSOCIATED WITH A BOX 


\title{
ON TWO POLYNOMIAL SPACES ASSOCIATED WITH A BOX SPLINE
}

\author{
CARl de Boor, Nira Dyn, and Amos Ron
}

\begin{abstract}
The polynomial space $\mathscr{H}$ spanned by the integer translates of a box spline $M$ admits a well-known characterization as the joint kernel of a set of homogeneous differential operators with constant coefficients. The dual space $\mathscr{H}^{*}$ has a convenient representation by a polynomial space $\mathscr{P}$, explicitly known, which plays an important role in box spline theory as well as in multivariate polynomial interpolation.

In this paper we characterize the dual space $\mathscr{P}$ as the joint kernel of simple differential operators, each one a power of a directional derivative. Various applications of this result to multivariate polynomial interpolation, multivariate splines and duality between polynomial and exponential spaces are discussed.
\end{abstract}

1. Introduction. The space $H(\phi)$ of all exponentials in the linear span $S(\phi)$ of the integer translates of a compactly supported distribution $\phi$ is of basic importance in multivariate spline theory since, in principle, it allows the construction of good approximation maps to $S(\phi)$ from spaces containing $S(\phi)$. Generically, $H(\phi)$ is $D$-invariant (i.e., closed under differentiation), hence is the joint kernel for a set $\left\{p(D): p \in I_{H(\phi)}\right\}$ of differential operators with constant coefficients, with $I_{H(\phi)}$ a polynomial ideal of finite codimension (in the space $\Pi$ of all multivariate polynomials, i.e., an ideal of transcendental dimension 0 , hence with finite variety). An understanding of the interplay between the space $H(\phi)$ and its associated ideal $I_{H(\phi)}$ is useful in the determination of the basic properties of $H(\phi)$ such as its spectrum, its dimension, and its local approximation order.

For the important special case when $\phi$ is a polynomial box spline (and $\mathscr{H}:=H(\phi)$ is thus a polynomial space), an explicit set of generators for the ideal $I_{\mathscr{H}}$ is known [BH1], but nevertheless, the construction of their joint kernel was found to be very difficult. At the same time, a polynomial space $\mathscr{P}$ (of very simple structure) is known which serves as a natural dual for $\mathscr{H}$ and is of substantial use in the analysis of $\mathscr{H}$. Specifically, the duality between $\mathscr{H}$ and $\mathscr{P}$ has been used in [DR1] in the investigation of the local approximation order of some exponential spaces, in [DR1,2] in the solution of an 
interpolation problem induced by $\mathscr{H}$, in $[\mathbf{J}]$ in the construction of linear projectors onto a box spline space, and in [DR1] in the computation of the homogeneous degrees of $\mathscr{H}$ (which is equivalent to computing the Hilbert function of $\left.I_{\mathscr{L}}\right)$. See also [DM3, §3].

It is the purpose of this paper to establish the surprising result that $\mathscr{P}$, too, is the joint kernel of a rather simple set of constant coefficient differential operators, each being just a power of a directional derivative. This allows us to characterize $\mathscr{P}$ in terms of the degrees of its polynomials when restricted to certain linear manifolds. Various applications of this result to multivariate polynomial interpolation, box spline theory, and duality between polynomial and exponential spaces are discussed as well.

In $\S 2$, after defining the space $\mathscr{P}$ and its associated differential operators, we prove that $\mathscr{P}$ is indeed the joint kernel of these operators. In $\S 3$, we identify $\mathscr{P}$ as a space of least degree among all polynomial spaces that interpolate correctly on certain subsets of the integer lattice. As a matter of fact, the discussion in that section may have an independent value: this discussion illustrates how the interpolating space of least degree from [BR1] may be computed using the technique from [BR2] of "perturbing the generators of a homogeneous ideal", hence in a computationally painless way.

Section 4 is devoted to the more general discussion of duality between finite-dimensional polynomial and exponential spaces, a discussion which improves proofs and results from [DM2] and [DR1]. Finally, we discuss in $\S 5$ the construction of piecewise polynomials on the support of a box spline and improve thereby an observation in [R].

2. The main result. Let $X$ be a multiset of vectors in $\mathbb{R}^{s} \backslash 0$. We will at times think of $X$, equivalently, as a real matrix, of size $(s \times \# X)$. Let $\mathbb{H}(X)$ denote the collection of all hyperplanes (i.e., linear subspaces of codimension 1) which are spanned by some columns of $X$. We associate with each $h \in \mathbb{H}(X)$ a nontrivial linear polynomial which vanishes on $h$, and write this polynomial

$$
\left\langle h^{\perp}, \cdot\right\rangle \text {, }
$$

thus using $h^{\perp}$ to stand for any particular nonzero vector normal to $h$. We are interested in the ideal $I^{X}$ generated by all polynomials of the form

$$
p_{h}:=p_{h, X}:=\left\langle h^{\perp}, \cdot\right\rangle^{\#(X \backslash h)}, \quad h \in \mathbb{H}(X),
$$

where $X \backslash h:=\{x \in X \mid x \notin h\}$. 
We assume that $X$ spans all of $\mathbb{R}^{s}$. Then the only point common to all $h \in \mathbb{H}(X)$ is 0 , and consequently, the variety of $I^{X}$ (i.e., the set of common zeros of all the polynomials in $I^{X}$ ) consists of 0 alone:

$$
\mathscr{V}_{I^{X}}=\{0\} \text {. }
$$

This implies that the codimension of $I^{X}$ in the space $\Pi$ of all polynomials in $s$ variables (i.e., the dimension of the quotient space $\Pi / I^{X}$ ) is finite, and that its kernel $I^{X} \perp$ is a finite-dimensional polynomial space, whose dimension equals the codimension of $I^{X}$. Moreover, $I^{X} \perp$ is stratified, i.e., spanned by its homogeneous elements, since $I^{X}$ is generated by homogeneous polynomials. [BR2] is a ready reference for these known facts.

Here, to recall the definition, the kernel $I \perp$ of an ideal $I$ is the set

$$
\left\{f \in \mathscr{D}^{\prime}\left(\mathbb{R}^{s}\right): p(D) f=0, \forall p \in I\right\}
$$

of all distributions annihilated by the set of differential operators induced by $I$. In particular, since $I^{X}$ is generated by

$$
p_{h}=\left\langle h^{\perp}, \cdot\right\rangle^{\#(X \backslash h)}, \quad h \in \mathbb{H}(X),
$$

$I^{X} \perp$ consists of the solutions $f$ of the system of linear differential equations

$$
\left(D_{h^{\perp}}\right)^{\#(X \backslash h)} f=0, \quad \forall h \in \mathbb{H}(X) .
$$

This section is devoted to a proof of the fact that $I^{X} \perp$ equals the polynomial space

$$
\mathscr{P}(X):=\operatorname{span}\left\{p_{V}: V \subset X, \operatorname{span}(X \backslash V)=\mathbb{R}^{S}\right\},
$$

with

$$
p_{V}:=\prod_{v \in V}\langle v, \cdot\rangle
$$

In the proof, the multiset

$$
\mathbb{B}(X):=\{B \subset X: B \text { invertible }\}
$$

of all bases contained in $X$ plays an important role. We use the abbreviation

$$
b(X):=\# \mathbb{B}(X) .
$$

(2.7) THEOREM. The kernel $I^{X} \perp$ of $I^{X}$ coincides with $\mathscr{P}(X)$, and

$$
\operatorname{dim} I^{X} \perp=b(X) \text {. }
$$

The theorem follows from the following three lemmata. 
(2.9) LEMMA.

$$
b(X) \leq \operatorname{dim} \mathscr{P}(X)
$$

(2.10) LEMMA.

$$
\mathscr{P}(X) \subset I^{X} \perp
$$

(2.11) LEMMA.

$$
\operatorname{dim} I^{X} \perp \leq b(X) .
$$

Proof [DR1] of (2.9) Lemma. Every polynomial

$$
q_{B}:=\prod_{x \in X \backslash B}\left(\langle x, \cdot\rangle-\lambda_{x}\right),
$$

with $B \in \mathbb{B}(X)$ and $\left(\lambda_{x}\right)$ arbitrary constants, is in $\mathscr{P}(X)$, as follows readily by multiplying out. For each $B \in \mathbb{B}(X)$, there is a unique common zero of the $s$ linear factors $\langle x, \cdot\rangle-\lambda_{x}, x \in B$, which do not occur in the associated $q_{B}$; call that point $\theta_{B}$. Choose now, as we may, the constants $\left(\lambda_{x}\right)$ in such a way that $\theta_{B} \neq \theta_{B^{\prime}}$ whenever $B \neq B^{\prime}$. (In fact, almost every choice of the $\lambda_{x}$ would satisfy this condition.) It then follows that

$$
q_{B}\left(\theta_{B^{\prime}}\right)=0 \Leftrightarrow B \neq B^{\prime},
$$

proving the linear independence of the collection $\left(q_{B}\right)_{B \in \mathbb{B}(X)}$ in $\mathscr{P}(X)$.

Proof of (2.10) Lemma. We have to prove that, for each $h \in \mathbb{H}(X)$, $p_{h}(D)=\left(D_{h^{\perp}}\right)^{\#(X \backslash h)}$ annihilates $\mathscr{P}(X)$, i.e., that $p_{h}(D) p_{V}=0$ for every $V \subset X$ for which $X \backslash V$ contains a basis. For this, we note that $D_{h^{\perp}} p_{V \cap h}=0$; hence

$$
p_{h}(D) p_{V}=\left(p_{V \cap h}\right) p_{h}(D) p_{V \backslash h} .
$$

On the other hand, since $X \backslash V$ contains a basis, $V \backslash h$ cannot coincide with $X \backslash h$, hence $\operatorname{deg} p_{V \backslash h}<\# X \backslash h=\operatorname{deg} p_{h}$ and therefore $p_{h}(D) p_{V \backslash h}=0$.

Proof of (2.11) Lemma. We prove the lemma by induction on \#X. For the case $\# X=s$ we observe that $I^{X}$ is generated by $s$ linearly independent linear homogeneous polynomials, consequently $I^{X} \perp$ contains only constants, and so $\operatorname{dim} I^{X} \perp=1=b(X)$. Assume now that $\# X>s$. We follow the argument used in the proof of [DM3; Thm. 3.1], decompose $X$ as

$$
X=: X^{\prime} \cup x
$$


with $\operatorname{span} X^{\prime}=\mathbb{R}^{s}$, and consider the map

$$
T: I^{X} \perp \rightarrow \underset{h \in \mathbb{H}\left(X^{\prime}\right)}{\times} P_{h}: q \mapsto\left(p_{h}(D) q\right)_{h \in \mathbb{H}\left(X^{\prime}\right)},
$$

where $p_{h}:=p_{h, X^{\prime}}=\left\langle h^{\perp}, \cdot\right\rangle^{\#\left(X^{\prime} \backslash h\right)}$ are the generators of $I^{X^{\prime}}$ and $P_{h}:=p_{h}(D)\left(I^{X} \perp\right)$. Then

$$
\operatorname{dim} I^{X} \perp \leq \operatorname{dim} I^{X^{\prime}} \perp+\sum_{h \in \mathbb{H}\left(X^{\prime}\right)} \operatorname{dim} P_{h},
$$

since

$$
\operatorname{ker} T=\left(I^{X^{\prime}} \perp\right) \cap\left(I^{X} \perp\right) \subseteq I^{X^{\prime}} \perp .
$$

Consequently, by induction,

$$
\operatorname{dim}\left(I^{X} \perp\right) \leq b\left(X^{\prime}\right)+\sum_{h \in \mathbb{H}\left(X^{\prime}\right)} \operatorname{dim} P_{h} .
$$

This proves that

$$
\operatorname{dim} I^{X} \perp \leq b(X),
$$

provided we can prove that

$$
\sum_{h \in \mathbb{H}\left(X^{\prime}\right)} \operatorname{dim} P_{h} \leq \#\{B \in \mathbb{B}(X): x \in B\} .
$$

In particular, it is sufficient to prove that, for all $h \in \mathbb{H}\left(X^{\prime}\right)$,

$$
P_{h} \subset I^{X_{h}} \perp
$$

with

$$
X_{h}:=(X \cap h) \cup x .
$$

For, (2.12) implies that

$$
\operatorname{dim} P_{h} \leq \operatorname{dim} I^{X_{h}} \perp \leq b\left(X_{h}\right),
$$

(the last inequality by induction), while

$$
\sum_{h \in \mathbb{H}\left(X^{\prime}\right)} b\left(X_{h}\right)=\#\{B \in \mathbb{B}(X): x \in B\} .
$$

The claim (2.12) is trivial in case $x \in h$, since then $X^{\prime} \backslash h=X \backslash h$, and therefore $p_{h, X^{\prime}}=p_{h, X}$ and so $P_{h}=\{0\}$ in that case.

We now prove (2.12) for the contrary case, i.e., the case when $x \notin$ $h$. We have to show that for every $k \in \mathbb{H}\left(X_{h}\right)$

$$
p_{k, X_{h}}(D) P_{h}=\{0\},
$$


with

$$
p_{k, X_{h}}=\left\langle k^{\perp}, \cdot\right\rangle^{\#\left(X_{h} \backslash k\right)} .
$$

If $x \notin k$, then $k=h$ and (since $\left.X_{h} \backslash h=\{x\}\right) p_{k, X_{h}}$ is a linear polynomial, and there is nothing to prove since then $p_{k, X_{h}} p_{h, X^{\prime}}=$ $p_{h, X}$, while $p_{h, X}$ annihilates $I^{X} \perp$ by definition of $I^{X} \perp$. For the contrary case that $x \in k$, we need to prove that

$$
D_{k^{\perp}}^{m} D_{h^{\perp}}^{A-m}\left(I^{X} \perp\right)=0,
$$

with

$$
m:=\#\left(X_{h} \backslash k\right), \quad A-m:=\#\left(X^{\prime} \backslash h\right) .
$$

For this, it is sufficient to show that

$$
K^{m} H^{A-m} \in \operatorname{ideal}\left\{L^{a(l)}\right\}_{l},
$$

with $l$ running over all hyperplanes spanned by elements of $X$ and containing $k \cap h$, and with

$$
\begin{aligned}
K:=\left\langle k^{\perp}, \cdot\right\rangle, \quad H:=\left\langle h^{\perp}, \cdot\right\rangle, \quad L:=\left\langle l^{\perp}, \cdot\right\rangle, \\
a(l):=\#(X \backslash l) .
\end{aligned}
$$

For, (2.14) follows from (2.15) since each generator $L^{a(l)}$ of the ideal in (2.15) appears also in the defining set (2.1) of generators of $I^{X}$, hence annihilates $I^{X} \perp$.

We prove $(2.15)$ by writing each polynomial $L$ as a linear combination of the linear polynomials $K$ and $H$, thereby obtaining the general homogeneous element of degree $A$ of the ideal in (2.15) in the form

$$
\sum_{l}(\alpha K+\beta H)^{a(l)} r_{l},
$$

with $r_{l}$ a homogeneous polynomial of degree

$$
\mu(l):=A-a(l),
$$

all $l$. We then show that the resulting linear system (for the coefficients of the various $r_{l}$ ) has a solution by showing that its coefficient matrix is the transpose of the matrix which occurs in (univariate polynomial) Hermite interpolation.

Here are the details.

Since each $l$ contains $k \cap h, l^{\perp}$ can be written uniquely as a linear combination of $k^{\perp}$ and $h^{\perp}$. We find it convenient in the sequel to have the weights in this linear combination sum to 1 , i.e., to have $l^{\perp}$ in the affine hull of $k^{\perp}$ and $h^{\perp}$. This we can achieve by first 
choosing the (signed) magnitudes of the nonzero vectors $k^{\perp}$ and $h^{\perp}$ so that their difference is not perpendicular to any of the finitely many $l$. Then, for each $l$, we choose the nonzero vector $l^{\perp}$ to lie on the line through $k^{\perp}$ and $h^{\perp}$, i.e., so that

$$
l^{\perp}=:(1-\beta) k^{\perp}+\beta h^{\perp}
$$

for some $\beta=\beta_{l}$. Then $L=(1-\beta) K+\beta H$; hence

$$
L^{a}=\sum_{j} K^{j} H^{a-j} B_{j}^{a}(\beta),
$$

with

$$
B_{j}^{r}(t):=\left(\begin{array}{l}
r \\
j
\end{array}\right)(1-t)^{j} t^{r-j}
$$

the polynomials appearing in the Bernstein form. Since $D B_{j}^{r}=$ $r\left(B_{j}^{r-1}-B_{j-1}^{r-1}\right)$, we have more generally

$$
((a+i) ! / a !)(H-K)^{i} L^{a}=\sum_{j} K^{j} H^{a+i-j} D^{i} B_{j}^{a+i}(\beta) .
$$

This means that we have available in our ideal an expression of the form

$$
\sum_{l} \sum_{i=0}^{\mu(l)}(H-K)^{i} L^{A-i} c(l, i)=\sum_{j} K^{j} H^{A-j} \sum_{l} \sum_{i=0}^{\mu(l)} D^{i} B_{j}^{A}(\beta) c(l, i)
$$

to match the monomial $K^{m} H^{A-m}$. Such a match is possible provided the linear system of conditions imposed thereby on the coefficients $c(l, i)$ is solvable.

We begin the proof that this linear system is indeed solvable by showing that its coefficient matrix is square. With the abbreviation $k^{\prime}:=h \cap k$, we compute

$$
m=\#\left(X_{h} \backslash k\right)=\#\left((X \cap h) \backslash k^{\prime}\right)=\#\left(\left(X^{\prime} \cap h\right) \backslash k^{\prime}\right),
$$

and therefore

$$
A=\#\left(\left(X^{\prime} \cap h\right) \backslash k^{\prime}\right)+\#\left(X^{\prime} \backslash h\right)=\#\left(X^{\prime} \backslash k^{\prime}\right) .
$$

Also,

$$
\begin{aligned}
\mu(l) & =A-a(l)=\#\left(X^{\prime} \backslash k^{\prime}\right)-\#(X \backslash l)=-1+\#\left(X \backslash k^{\prime}\right)-\#(X \backslash l) \\
& =\#\left((X \cap l) \backslash k^{\prime}\right)-1 .
\end{aligned}
$$

Therefore, the number of unknowns is

$$
\sum_{l}(\mu(l)+1)=\sum_{l} \#\left((X \cap l) \backslash k^{\prime}\right)=\#\left(X \backslash k^{\prime}\right)=A+1,
$$


i.e., equal to the number of equations. Here, the sums are over all $l \in \mathbb{H}(X)$ which contain $k^{\prime}$, which implies that $X \backslash k^{\prime}$ is the disjoint union of the sets $(X \cap l) \backslash k^{\prime}$ and so justifies the second equality.

Now organize the unknowns by $l$ and, within $l$, by $i=0, \ldots, \mu(l)$, and order the equations by $j=0, \ldots, A$. Then the matrix consists, more precisely, of one block of columns for each $l$, with the $i$ th column (in the block for $l$ ) containing the value at $\beta=\beta_{l}$ of the $i$ th derivative of all the polynomials $B_{j}^{A}, j=0, \ldots, A, i=0, \ldots, \mu(l)$. Hence our matrix is the transpose of the matrix which occurs in the linear system for the determination of the Bernstein form of the polynomial in $\Pi_{A}$ which agrees with some function $(\mu(l)+1)$-fold at $\beta=\beta_{l}$, all $l$. Since such univariate Hermite interpolation is correct (since $\beta_{l} \neq \beta_{l^{\prime}}$ for $l \neq l^{\prime}$ ), the invertibility of our matrix follows.

We note that (2.7) Theorem now allows us to conclude that all inequalities appearing in its proof must be equalities. This implies, e.g., that

$$
I^{X^{\prime}} \subset I^{X} \text { whenever } X^{\prime} \subset X,
$$

and that $p_{h, X^{\prime}}(D)\left(I^{X} \perp\right)=I^{h \cup x} \perp$. Another immediate consequence is the following

(2.17) CoRollaRY [DM3], [DR1].

$$
\operatorname{dim} \mathscr{P}(X)=b(X) \text {. }
$$

Furthermore,

$$
d(X):=\min \{\#(X \backslash h): h \in \mathbb{H}(X)\}
$$

is the least degree of the generators of $I^{X}$; hence, since $I^{X} \perp=\mathscr{P}(X)$, we have the following.

(2.19) CoRollaRY [DR1]. With $d(X)$ as in (2.18),

$$
\Pi_{<d(X)} \subset \mathscr{P}(X),
$$

but

$$
\Pi_{d(X)} \not \subset \mathscr{P}(X) \text {. }
$$

By its definition, $I^{X} \perp$ is the set of all polynomials $p \in \Pi$ that satisfy the following condition:

(2.20) Condition. For every $h \in \mathbb{H}(X)$ and $v \in \mathbb{R}^{s},\left.p\right|_{v+h \perp} \subset$ $\Pi_{\#(X \backslash h)-1}$ (with $h \perp$ the subspace orthogonal to $h$ ). 
On the other hand, every $p \in \mathscr{P}(X)$ satisfies an apparently stronger condition (cf. [DR2; Prop.1]):

(2.21) Condition. For every subspace $M$ of $\mathbb{R}^{s}$ which is spanned by elements from $X$, and for every $v \in \mathbb{R}^{s}$,

$$
\left.p\right|_{v+M \perp} \in \Pi_{\#(X \backslash M)-\operatorname{dim} M \perp} .
$$

Hence we conclude the following from (2.7) Theorem:

(2.22) Corollary. The space $\mathscr{P}(X)$ is characterized either by (2.20) Condition or by (2.21) Condition. In particular, these two conditions are equivalent on $\Pi$.

(2.22) Corollary verifies the claim made in [DR2; Remark 2] that (2.21) Condition characterizes $\mathscr{P}(X)$. In case $X$ consists of $N$ repetitions of $s+1$ vectors $Y \subset \mathbb{R}^{s}$ in general position, the characterization of $\mathscr{P}(X)$ by (2.21) Condition has been proved in [G] by other methods.

3. An associated polynomial interpolation problem. Here we identify certain exponential spaces $H$ whose corresponding "limit at the origin" $H_{\downarrow}$ coincides with $\mathscr{P}(X)$ (for an appropriate choice of $X$ ), and use this identification in the solution of an associated interpolation problem. The map

$$
H \mapsto H_{\downarrow},
$$

which associates with every finite-dimensional space of entire functions a homogeneous space of polynomials of the same dimension, has been introduced and studied in [BR1] in the context of a multivariate polynomial interpolation problem, and has been discussed as well in [BR2] in the context of kernels of polynomial ideals. To begin with, we recall the definition of $H_{\downarrow}$ and review some of the results of [BR1, 2] needed here. Then we discuss a certain interpolation problem and its relation to $\mathscr{P}(X)$.

Given a function $f \neq 0$ analytic at the origin, we write its power series expansion at the origin in the form

$$
f=f_{0}+f_{1}+f_{2}+\cdots,
$$

where, for each $j, f_{j}$ is a homogeneous polynomial of degree $j$, and define $f_{\downarrow}:=f_{k}$ with $k=\min \left\{j: f_{j} \neq 0\right\}$; i.e., $f_{\downarrow}$ is the first nontrivial homogeneous polynomial in the power expansion of $f$. Using 
this notion of the least term $f_{\downarrow}$ of $f$, we then define

$$
H_{\downarrow}:=\operatorname{span}\left\{f_{\downarrow}: f \in H\right\},
$$

and have [BR1]

$$
\operatorname{dim} H_{\downarrow}=\operatorname{dim} H .
$$

We say that a pointset $\Theta \subset \mathbb{R}^{S}$ is correct for the polynomial space $P$ if the restriction map $P \rightarrow \mathbb{C}^{\Theta}:\left.p \mapsto p\right|_{\Theta}$ is invertible. Equivalently, $\Theta$ is correct for $P$ if, for every data $\left(d_{\theta}\right)_{\theta \in \Theta}$, there is exactly one $p \in P$ for which $p(\theta)=d_{\theta}$ for all $\theta \in \Theta$. In other words, interpolation from $P$ at the points of $\Theta$ is correct.

RESULT [BR1]. $\Theta$ is correct for $\left(\exp _{\Theta}\right)_{\downarrow}$. Moreover, among all polynomial spaces $P$ for which $\Theta$ is correct, $\left(\exp _{\Theta}\right)_{\downarrow}$ is "of least degree" in the sense that

$$
\operatorname{dim}\left(P \cap \Pi_{j}\right) \leq \operatorname{dim}\left(\left(\exp _{\Theta}\right)_{\downarrow} \cap \Pi_{J}\right), \quad \forall j .
$$

Here,

$$
\exp _{\Theta}:=\operatorname{span}\left\{e_{\theta}\right\}_{\theta \in \Theta},
$$

where $e_{\theta}: x \mapsto e^{\langle\theta, x\rangle}$ is the exponential function (with frequency $\theta$ ).

In view of this result, it is useful to be able to identify the "least" space $\left(\exp _{\Theta}\right)_{\downarrow}$ for given $\Theta$. This we now do for certain pointsets $\Theta=\nu_{X}$ associated with the box splines $M_{X}$. Our tool is the following result

\section{(3.6) Result [BR2].}

$$
H_{\downarrow}=\left(I_{H \uparrow}\right) \perp
$$

which obtains $H_{\downarrow}$ as the kernel of the ideal generated by the leading terms of the annihilator of $H$. Precisely, [BR2; (4.3) Theorem (b)] provides the statement that, for any polynomial ideal I of finite codimension,

$$
(I \perp)_{\downarrow}=\left(I_{\uparrow}\right) \perp,
$$

and (3.6) Result is obtained by applying this to

$$
I=I_{H}:=\{p \in \Pi: p(D) H=0\},
$$

which is an ideal since $H$ is closed under differentiation. In fact, with $H=\exp _{\Theta}, I_{H}$ consists of all polynomials which vanish at $\Theta$; hence $I_{H}$ has finite codimension and

$$
H=I_{H} \perp,
$$


(cf. [BR2, §3]). From $I_{H}$, we obtain its homogeneous counterpart $I_{H \uparrow}$ as

$$
I_{H \uparrow}:=\operatorname{span}\left\{p_{\uparrow}: p \in I_{H}\right\},
$$

where $p_{\uparrow}$ is the leading term of the polynomial $p$, namely the homogeneous polynomial satisfying

$$
\operatorname{deg}\left(p-p_{\uparrow}\right)<\operatorname{deg} p .
$$

The result (3.6) is of interest here since it is easy to identify elements of $I_{H}$ in case $H=\exp _{\Theta}$ : If $\left(p_{j}\right)$ are linear homogeneous polynomials for which the union of the corresponding hyperplanes $\left\{x \in \mathbb{R}^{s}: p_{j}(x)=c_{j}\right\}$ (for suitable choices of the constants $c_{j}$ ) contains $\Theta$, then $p:=\prod_{j}\left(p_{j}-c_{j}\right) \in I_{H}$; hence $\prod_{j} p_{j} \in\left(I_{H}\right)_{\uparrow}$. If we obtain enough of these $p$ to generate all of $I_{H \uparrow}$, then we know by (3.6) Result that $H_{\downarrow}$ is the joint kernel of all the corresponding differential operators $p(D)$. In fact, since we know from (3.3) that $\operatorname{dim} H_{\downarrow}=\operatorname{dim} H$, we can already reach this conclusion when we only know that the $p$ so constructed generate an ideal $J$ of codimension $\leq \operatorname{dim} H$.

(3.9) RESULT [BR2]. If the ideal $J$ generated from the leading terms of some polynomials in $I_{H}$ has codimension $\leq \operatorname{dim} H$, then $J=I_{H \uparrow}$; therefore

$$
H_{\downarrow}=J \perp \text {. }
$$

In our case, we have identified (in (2.7) Theorem) $\mathscr{P}(X)$ as the joint kernel of the differential operators $\left(D_{h}\right)^{\#(X \backslash h)}$ (with $h$ running over $\mathbb{H}(X))$, hence are entitled to conclude that $\left(\exp _{\Theta}\right)_{\downarrow}=\mathscr{P}(X)$ whenever we can find, for each such $h$, constants $c_{j, h}$ so that

$$
\prod_{j=1}^{\#(X \backslash h)}\left(\left\langle h^{\perp}, \cdot\right\rangle-c_{j, h}\right)
$$

vanishes on $\Theta$, and know additionally that $\# \Theta \geq \operatorname{dim} \mathscr{P}(X)$.

Just such a pointset is, under certain assumptions on $X$, provided by

(3.10) $\nu_{X}:=\nu_{X}(z):=\left\{\alpha \in \mathbb{Z}^{s}: z-\alpha=\sum_{x \in X} t_{x} x, 0<t_{x}<1, \forall x\right\}$,

with $z \in \mathbb{R}^{S} \backslash \bigcup_{h \in \mathbb{H}(X)}\left(h+\mathbb{Z}^{s}\right)$. The set $-\nu_{X}$ comprises the integer points in the support of the shifted box spline $M_{X}(\cdot+z)$ (cf. [DM2]). 
Assume now that $X$ is unimodular, i.e., the columns of $X$ are from $\mathbb{Z}^{S} \backslash 0$ and every $B \in \mathbb{B}(X)$ has determinant \pm 1 . For such unimodular $X$, the observations made in [R; $\S 4]$ (especially before the proof of Theorem 4.1 and in the proof of Corollary 4.2) confirm the existence, for each $h \in \mathbb{H}(X)$, of consecutive integers $c_{h, j}$ so that $\nu_{X}$ is contained in the union of the hyperplanes

$$
\left\langle h^{\perp}, \cdot\right\rangle=c_{h, j}, \quad j=1, \ldots, \# X \backslash h .
$$

Indeed, fixing $h \in \mathbb{H}(X)$ and taking the normal $h^{\perp}$ to be a relatively prime integer vector implies that $\left|\left\langle h^{\perp}, x\right\rangle\right|=1$ for every $x \in X \backslash h$ since $X$ is unimodular. By choosing the signs of the columns of $X$ appropriately (which amounts to a shift in $M_{X}$ ), we can achieve that $\left\langle h^{\perp}, x\right\rangle>0$ for all $x \in X \backslash h$; hence

$$
c_{h}:=\sum_{x \in X \backslash h}\left\langle h^{\perp}, x\right\rangle=\# X \backslash h .
$$

Consequently, with $z$ chosen so as to satisfy $c_{h}-1<\left\langle h^{\perp}, z\right\rangle\left\langle c_{h}\right.$, $\nu_{X}(z)$ must lie in the union of the hyperplanes

$$
\left\langle h^{\perp}, \cdot\right\rangle=j, \quad j=0, \ldots, c_{h}-1 .
$$

Moreover, $\# \nu_{X}=\operatorname{dim} \mathscr{P}(X)$, because of (2.17) Corollary and the following.

(3.11) Result [DM2]. If $X$ is unimodular, then

$$
\# \nu_{X}=b(X) \text {. }
$$

This establishes the following theorem.

(3.12) THEOREM. If $X$ is unimodular, then $\left(\exp _{\nu_{X}}\right)_{\downarrow}=\mathscr{P}(X)$. In particular, $\nu_{X}$ is correct for $\mathscr{P}(X)$, and $\mathscr{P}(X)$ is of least degree among all polynomial spaces for which $\nu_{X}$ is correct.

Note that only the inequality $\# \nu_{X} \geq b(X)$ was needed in the proof of (3.12) Theorem. As a matter of fact, the converse inequality is a consequence of the theorem.

4. The duality between $\mathscr{H}(X)$ and $\mathscr{P}(X)$. The ideal $I^{X}$ and its kernel $\mathscr{P}(X)$ are intimately related to another ideal $I_{X}$ and its kernel $\mathscr{H}(X)$, which play a fundamental role in the theory of box splines. In the following, we review some of the basics about $I_{X}$ and $\mathscr{H}(X)$ and draw several connections between the two settings. 
Let $I_{X}$ be the ideal generated by all polynomials of the form $p_{V}=$ $\prod_{v \in V}\langle v, \cdot\rangle$ with $V \subset X$ and $\operatorname{span}(X \backslash V) \neq \mathbb{R}^{s}$. This means that

$$
I_{X}:=\operatorname{ideal}\left\{p_{X \backslash h}: h \in \mathbb{H}(X)\right\} .
$$

Let $\mathscr{H}(X)$ denote the kernel of $I_{X}$, i.e.,

$$
\mathscr{H}(X):=I_{X} \perp \text {. }
$$

It is known [BH1], [DM1] that $\mathscr{H}(X)$ is a finite-dimensional polynomial space and [DM2] that

$$
\operatorname{dim} \mathscr{H}(X)=b(X) .
$$

The spaces $\mathscr{P}(X)$ and $\mathscr{H}(X)$ are dual to each other in the following sense. Each $p \in \Pi$ gives rise to a linear functional $p^{*}$ on $\Pi$ (and even on a larger space of smooth functions), viz. the linear functional

$$
p^{*}: q \mapsto p(D) q(0) .
$$

This allows us to consider, for any two finite-dimensional linear polynomial spaces $Q$ and $R$, the map

$$
M: Q \rightarrow R^{*}:\left.p \mapsto p^{*}\right|_{R} .
$$

If $M$ is invertible, we say that $Q$ is dual to $R$ (in the sense that we can then use the elements of $Q$ in this fashion to represent uniquely the dual of $R$ ). Note that the dual to $M$ carries $R^{* *}=R$ in the same way to $Q^{*}$; hence $Q$ is dual to $R$ iff $R$ is dual to $Q$.

A necessary and sufficient condition for such duality is that $M$ be 1-1 and $\operatorname{dim} R \leq \operatorname{dim} Q$ (since then $M$ is necessarily onto). In particular, if $\operatorname{dim} Q=\operatorname{dim} R$, then such duality is assured as soon as we know that, for every $q \in Q \backslash 0$, there is an $r \in R$ for which $q^{*}(r) \neq 0$. By the duality already mentioned, this is equivalent to having, for every $r \in R \backslash 0$, a $q \in Q$ for which $q^{*}(r) \neq 0$.

For $q \in Q$, the linear functional $q^{*}$ cannot tell the difference between $f$ and $T_{k} f:=f_{0}+\cdots+f_{k-1}:=$ the power expansion of $f$ up to order $k$, if $k$ is sufficiently large. This allows us to extend this notion of duality to pairs $Q, R$ in which $R$ is a finite-dimensional space of smooth functions.

We will eventually make use of the following observation:

(4.3) Theorem. Let $P$ be an n-dimensional homogeneous polynomial space. Let $H$ be an n-dimensional space of entire functions. If $P$ is dual to $H_{\downarrow}$, then $P$ is dual to $H$.

Proof. For any $f \in H \backslash 0, f_{\downarrow} \in H_{\downarrow} \backslash 0$; hence, by assumption, $p^{*}\left(f_{\downarrow}\right)$ $\neq 0$ for some $p \in P$. Further, since $P$ is spanned by homogeneous 
polynomials, we may assume that this $p$ is homogeneous. But then $p^{*}(f)=p^{*}\left(f_{\downarrow}\right) \neq 0$, showing that the linear map $H \rightarrow P^{*}: f \mapsto$ $\left(p \mapsto p^{*}(D) f(0)\right)$ is $1-1$; hence $P \rightarrow H^{*}:\left.p \mapsto p^{*}\right|_{H}$ is onto. Since $\operatorname{dim} H=\operatorname{dim} H_{\downarrow}$, and $\operatorname{dim} H_{\downarrow}=\operatorname{dim} P$ by assumption, the theorem follows.

We note that the converse of (4.3) Theorem does not hold, in general. For, it is easy to make up a nonhomogeneous polynomial space $H$ together with a homogeneous $P$ dual to it, for which the conditions $\operatorname{dim}\left(\Pi_{j} \cap P\right)=\operatorname{dim}\left(\Pi_{j} \cap H_{\downarrow}\right)$, all $j$, fail to hold, while these conditions are necessary for $P$ and $H_{\downarrow}$ to be dual, according to the following proposition of use later.

(4.4) Proposition. If the homogeneous polynomial spaces $Q$ and $R$ are dual to each other, then

$$
\operatorname{dim}\left(\Pi_{j} \cap Q\right)=\operatorname{dim}\left(\Pi_{j} \cap R\right)
$$

for all $j$.

Proof. Indeed, if (4.5) is violated for some (minimal) $j$ and, say, $\operatorname{dim}\left(\Pi_{j} \cap Q\right)>\operatorname{dim}\left(\Pi_{j} \cap R\right)$, then there exists a homogeneous polynomial $q \in Q$ of degree $j$ for which $q^{*}$ vanishes on all homogeneous polynomials in $R$ of degree $j$, and hence vanishes on all of $R$, in contradiction to the duality between $Q$ and $R$.

With this, the meaning of the following result is clear.

(4.6) Result [DM3]', [DR1]. The polynomial spaces $\mathscr{P}(X)$ and $\mathscr{H}(X)$ are dual to each other.

In (3.12) Theorem, the space $\mathscr{P}(X)$ has been identified as the least space for certain interpolation problems. In [BR2] the space $\mathscr{H}(X)$ has been identified as the least space for other interpolation problems. We now make use of the duality between $\mathscr{P}(X)$ and $\mathscr{H}(X)$ to connect $\mathscr{H}(X)$ with the interpolation problems associated with $\mathscr{P}(X)$ and vice versa. As a preparation, we procure a class of spaces whose corresponding least space is $\mathscr{H}(X)$ in much the same way in which we obtained suitable exponential spaces $\exp _{\nu_{X}}$ whose least is $\mathscr{P}(X)$ : We perturb the linear factors of the set of generators for the

\footnotetext{
${ }^{1}$ The authors in [DM3] attribute the result to Hakopian.
} 
ideal (viz. $I_{X}$ ) whose kernel is $\mathscr{H}(X)$. Specifically, given any map $\Gamma: X \rightarrow \mathbb{C}: x \mapsto \Gamma_{x}$, we consider the ideal

$$
I_{\Gamma}:=\operatorname{ideal}\left\{q_{h}: h \in \mathbb{H}(X)\right\},
$$

with

$$
q_{h}:=\prod_{x \in X \backslash h}\left(\langle x, \cdot\rangle-\Gamma_{x}\right) .
$$

Then $\mathscr{H}(\Gamma):=I_{\Gamma} \perp$ is an exponential space (i.e., a space which is spanned by certain products of exponentials with polynomials), and [BR2]

$$
\mathscr{H}(\Gamma)_{\downarrow}=\mathscr{H}(X) .
$$

The diagram below illustrates for a unimodular $X$ the various connections established so far between the ideals $I^{X}, I_{X}$, their kernels and the associated exponential spaces.

$$
\begin{array}{cc}
\left(\exp _{\nu_{X}}\right)_{\downarrow}= & \mathscr{P}(X)=I^{X} \perp \\
& \text { dual } \\
\mathscr{H}(\Gamma)_{\downarrow}= & \stackrel{\downarrow}{ }(X)=I_{X} \perp
\end{array}
$$

Our first corollary improves [DM2; Thm. 4.1]:

(4.9) CoRollary. Let $X$ be unimodular. Then $\nu_{X}$ is correct for $\mathscr{H}(X)$, and $\mathscr{H}(X)$ is of least degree among all polynomial spaces for which $\nu_{X}$ is correct.

Proof. We apply (4.3) Theorem with $H=\exp _{\nu_{x}}$ and $P=\mathscr{H}(X)$. By (3.12) Theorem, $H_{\downarrow}=\mathscr{P}(X)$, while by (4.6) Result, $\mathscr{P}(X)$ is dual to $\mathscr{H}(X)$. Since also $\mathscr{H}(X)$ is homogeneous (as the kernel of a homogeneous ideal), (4.3) Theorem implies that $\mathscr{H}(X)$ is dual to $\exp _{\nu_{X}}$, which is equivalent (cf. [BR1; $\left.\S 4\right]$ ) to the correctness of $\nu_{X}$ for $\mathscr{H}(X)$. Moreover, since $\mathscr{P}(X)$ and $\mathscr{H}(X)$ are both homogeneous and, by (4.6) Result, dual to each other, we must have

$$
\operatorname{dim}\left(\Pi_{j} \cap \mathscr{H}(X)\right)=\operatorname{dim}\left(\Pi_{j} \cap \mathscr{P}(X)\right)
$$

for every $j$, by (4.4) Proposition. Since we already know by (3.12) Theorem that $\mathscr{P}(X)$ is of least degree among all polynomial spaces for which $\nu_{X}$ is correct, it follows from (4.10) that $\mathscr{H}(X)$ has the same property. 
(4.11) CoRollaRY [DR1]. The spaces $\mathscr{P}(X)$ and $\mathscr{H}(\Gamma)$ are dual to each other.

Proof. Take $P=\mathscr{P}(X)$ and $H=\mathscr{H}(\Gamma)$ in (4.3) Theorem. Since, by $(4.8), \mathscr{H}(\Gamma)_{\downarrow}=\mathscr{H}(X)$, and , by (4.6) Result, $\mathscr{H}(X)$ is dual to $\mathscr{P}(X),(4.3)$ Theorem provides the desired result.

We refer to [DR1; $\S 7]$ for a discussion of the interpolation conditions induced by $\mathscr{H}(\Gamma)$.

5. Application to box splines. In this section we point out some connections between the results of the previous sections and the theory of multivariate splines. In the discussion here the (polynomial) box spline $M_{X}$ associated with a set of directions $X$ plays a central role. For our purposes, it is sufficient to note that $M_{X}$ is a piecewisepolynomial function supported on

$$
\Omega_{X}:=\left\{X t: t \in[0,1]^{\# X}\right\}
$$

and satisfies

$$
\Pi\left(M_{X}\right)=\mathscr{H}(X)
$$

where, for a general compactly supported $\phi$, the notation $\Pi(\phi)$ stands for the space of polynomials spanned by the integer translates of $\phi$.

We make use of the following result, which is a special case of [R; Thm.1.1]:

(5.1) RESULT. Let $P$ be a translation-invariant space of polynomials, and $\Omega$ a compact subset of $\mathbb{R}^{s}$ with boundary $\partial \Omega$. Then the following conditions are equivalent:

(a) There exists a function $\phi$ supported in $\Omega$ and satisfying $\Pi(\phi)=$ $P, \widehat{\phi}(0) \neq 0$.

(b) For every $z \in \mathbb{R}^{s} \backslash \bigcup_{\alpha \in \mathbb{Z}^{s}} \alpha+\partial \Omega$, the set

$$
\nu_{\Omega}:=\nu_{\Omega}(z):=\left\{\alpha \in \mathbb{Z}^{S}: z-\alpha \in \Omega\right\}
$$

is total for $P$, i.e., no element of $P \backslash 0$ vanishes on this set.

Note that when we take $\Omega$ to be $\Omega_{X}=\operatorname{supp} M_{X}$, the sets $\nu_{\Omega}$ are identical with the sets $\nu_{X}$ from (3.10). Thus, by appealing to (3.12) Theorem, we deduce from the implication (b) $\Rightarrow(a)$ of (5.1) Result 
the following

(5.2) COROLlARY. Let $X$ be a unimodular set of vectors. Then there exists a function (actually many) $\psi=\psi_{X}$ which is supported in $\Omega_{X}$ and satisfies

$$
\Pi(\psi)=\mathscr{P}(X)
$$

The above corollary provides no information about the smoothness of the compactly supported $\psi$. Yet, it is known [BH2] that, at least for the special case of the three-direction mesh with equal multiplicities, no piecewise- $\mathscr{P}(X)$ function supported on $\Omega_{X}$ can match the smoothness of the corresponding box spline $M_{X}$.

The box spline $M_{X}$ is a smooth function supported on $\Omega_{X}$. Hence, one may hope that there exist functions $\phi$ supported on $\Omega_{X}$ which are less smooth than $M_{X}$, yet their corresponding $\Pi(\phi)$ is "better" in the sense that it contains some of the polynomials of lower degrees which were missing in $\Pi\left(M_{X}\right)=\mathscr{H}(X)$. [R; Cor. 4.2] gives a partial negative answer to that hope by showing that for a unimodular $X$ and a function $\phi$ supported in $\Omega_{X}$, if $\widehat{\phi}(0) \neq 0$ and $\Pi_{j} \subset \Pi(\phi)$ for some $j$, then $\Pi_{j} \subset \mathscr{H}(X)=\Pi\left(M_{X}\right)$. The following result improves that corollary.

(5.3) Corollary. Let $X$ be a unimodular set of directions, $M_{X}$ the corresponding box spline. Let $\phi$ be a compactly supported function satisfying

$$
\operatorname{supp} \phi \subset \operatorname{supp} M_{X}
$$

and

$$
\widehat{\phi}(0) \neq 0
$$

Then, for each $j$,

$$
\operatorname{dim}\left(\Pi_{j} \cap \Pi(\phi)\right) \leq \operatorname{dim}\left(\Pi_{j} \cap \Pi\left(M_{X}\right)\right) .
$$

Proof. Let $\nu$ be one of the sets $\nu_{X}(z)$ associated with $X$. By (3.12) Theorem, $\left(\exp _{\nu}\right)_{\downarrow}=\mathscr{P}(X)$. Now we may apply (5.1) Result to conclude that $\nu$ is total for $\Pi(\phi)$, and hence $\Pi(\phi)$ can be extended to a space $Q$ for which $\nu$ is correct. Since $\mathscr{P}(X)$ is the least space of $\exp _{\nu}$, it satisfies the least degree property (3.4), thus we conclude that for every $j$

$$
\operatorname{dim}\left(\Pi_{j} \cap \Pi(\phi)\right) \leq \operatorname{dim}\left(\Pi_{j} \cap Q\right) \leq \operatorname{dim}\left(\Pi_{j} \cap \mathscr{P}(X)\right) .
$$


On the other hand, by (4.4) and (4.6),

$$
\operatorname{dim}\left(\Pi_{j} \cap \mathscr{P}(X)\right)=\operatorname{dim}\left(\Pi_{j} \cap \mathscr{H}(X)\right), \quad \forall j,
$$

and (5.3) now follows from the fact that $\Pi\left(M_{X}\right)=\mathscr{H}(X)$.

We note that the result is no longer valid if we drop the unimodularity assumption (cf. [R; Ex. 4.1]).

6. A remark on (2.11) Lemma. A careful examination of the proof of (2.11) Lemma shows that the details of the connection between the ideal $I^{X}$ and its kernel $I^{X} \perp$ enter into the argument in only a minor way. The only facts used are: (i) the map $\Pi \rightarrow L(\Pi): p \mapsto p(D)$ is a ring homomorphism; and (ii) for any basis $B$ in $X, \operatorname{dim} I^{B} \perp=1$.

This suggests the following result.

(6.1) Proposition. Let $M: \Pi \rightarrow L(V)$ be a ring-homomorphism into the ring of linear maps on the linear space $V$. For a given multiset $X$ of directions, define

$$
I_{M}^{X} \perp:=\bigcap_{p \in I^{X}} \operatorname{ker} M(p) .
$$

Then

$$
\operatorname{dim} I_{M}^{X} \perp \leq \sum_{B \in \mathbb{B}(X)} \operatorname{dim} I_{M}^{B} \perp
$$

The proof of the proposition follows entirely that of (2.11) Lemma, with the obvious modifications whenever the induction hypothesis is applied.

It would be nice to identify other settings rather than the one utilized in this paper, where the above proposition is of use.

\section{REFERENCES}

[BH1] C. de Boor and K. Höllig, B-splines from parallelepipeds, J. d'Anal. Math., $42(1982 / 3), 99-115$.

[BH2] _ Bivariate box splines and smooth pp functions on a three-direction mesh, J. Comput. Applied Math., 9 (1983), 13-28.

[BR1] C. de Boor and A. Ron, On multivariate polynomial interpolation, Construetive Approx., 6 (1990), 287-302.

[BR2] - On polynomial ideals of finite codimension with application to box spline theory, J. Math. Anal. Appl., to appear.

[DM1] W. Dahmen and C. A. Micchelli, Translates of multivariate splines, Linear Algebra and Appl., 52/3 (1983), 217-234. 
[DM2] W. Dahmen and C. A. Micchelli, On the local linear independence of translates of a box spline, Studia Math., 82 (1985), 243-263.

[DM3] _., Multivariate E-splines, Advances in Math., 76 (1989), 33-93.

[DR1] N. Dyn and A. Ron, Local approximation by certain spaces of multivariate exponential-polynomials, approximation order of exponential box splines and related interpolation problems, Trans. Amer. Math. Soc., 319 (1990), $381-$ 404.

[DR2] _ On multivariate polynomial interpolation, in Algorithms for Approximation II, J. C. Mason, M. G. Cox (eds.), Chapman and Hall, London 1990, 177-184.

[G] J. A. Gregory, Interpolation to boundary data on the simplex, CAGD, 2 (1985), 43-52.

[J] R. Q. Jia, A dual basis for the integer translates of an exponential box spline, preprint 1988.

[R] A. Ron, Relations between the support of a compactly supported function and the exponential-polynomials spanned by its integer translates, Constructive Approx., 5 (1989), 297-308.

Received April 17, 1989. The first author was supported by the National Science Foundation under Grant No. DMS-8701275 and by the United States Army under Contract No. DAAL03-87-K-0030.

UNIVERSITY OF WISCONSIN

MAdisON, WI 53706

TEL-AVIV UNIVERSITY

TEL-AVIV, ISRAEL

AND

UNIVERSITY OF WISCONSIN

MADISON, WI 53706 



\title{
PACIFIC JOURNAL OF MATHEMATICS EDITORS
}

\author{
V. S. VARADARAJAN \\ (Managing Editor) \\ University of California \\ Los Angeles, CA 90024-1555-05 \\ Herbert Clemens \\ University of Utah \\ Salt Lake City, UT 84112 \\ THOMAS ENRIGHT \\ University of California, San Diego \\ La Jolla, CA 92093
}

R. FINN

Stanford University

Stanford, CA 94305

Hermann FlaschKa

University of Arizona

Tucson, AZ 85721

VAUGHaN F. R. JoNes

University of California

Berkeley, CA 94720

Steven Kerckhoff

Stanford University

Stanford, CA 94305

\section{C. MOORE}

University of California

Berkeley, CA 94720

Martin ScharlemanN

University of California

Santa Barbara, CA 93106

HAROLD STARK

University of California, San Diego

La Jolla, CA 92093
R. ARENS
E. F. BECKENBACH
F. WolF
K. YoshidA

\section{ASSOCIATE EDITORS}

\begin{tabular}{ll}
\multicolumn{1}{c}{ SUD-1982) } & \multicolumn{1}{c}{$(1904-1989)$} \\
UNIVERSITY OF ARIZONA & UNIVERSITY OF OREGON \\
UNIVERSITY OF BRITISH COLUMBIA & UNIVERSITY OF SOUTHERN CALIFORNIA \\
CALIFORNIA INSTITUTE OF TECHNOLOGY & STANFORD UNIVERSITY \\
UNIVERSITY OF CALIFORNIA & UNIVERSITY OF HAWAII \\
MONTANA STATE UNIVERSITY & UNIVERSITY OF TOKYO \\
UNIVERSITY OF NEVADA, RENO & UNIVERSITY OF UTAH \\
NEW MEXICO STATE UNIVERSITY & WASHINGTON STATE UNIVERSITY \\
OREGON STATE UNIVERSITY & UNIVERSITY OF WASHINGTON \\
\hline
\end{tabular}

The Supporting Institutions listed above contribute to the cost of publication of this Journal, but they are not owners or publishers and have no responsibility for its content or policies.

\begin{abstract}
Mathematical papers intended for publication in the Pacific Journal of Mathematics should be in typed form or offset-reproduced (not dittoed), double spaced with large margins. Please do not use built up fractions in the text of the manuscript. However, you may use them in the displayed equations. Underline Greek letters in red, German in green, and script in blue. The first paragraph must be capable of being used separately as a synopsis of the entire paper. In particular it should contain no bibliographic references. Please propose a heading for the odd numbered pages of less than 35 characters. Manuscripts, in triplicate, may be sent to any one of the editors. Please classify according to the 1980 Mathematics Subject Classification (1985 Revision) scheme which can be found in the December index volumes of Mathematical Reviews. Supply name and address of author to whom proofs should be sent. All other communications should be addressed to the managing editor, or Elaine Barth, University of California, Los Angeles, California 90024-1555-05.

There are page-charges associated with articles appearing in the Pacific Journal of Mathematics. These charges are expected to be paid by the author's University, Government Agency or Company. If the author or authors do not have access to such Institutional support these charges are waived. Single authors will receive 50 free reprints; joint authors will receive a total of 100 free reprints. Additional copies may be obtained at cost in multiples of 50 .
\end{abstract}

The Pacific Journal of Mathematics (ISSN 0030-8730) is published monthly except for July and August. Regular subscription rate: $\$ 190.00$ a year (10 issues). Special rate: $\$ 95.00$ a year to individual members of supporting institutions.

Subscriptions, orders for numbers issued in the last three calendar years, and changes of address should be sent to Pacific Journal of Mathematics, P.O. Box 969, Carmel Valley, CA 93924, U.S.A. Old back numbers obtainable from Kraus Periodicals Co., Route 100, Millwood, NY 10546.

The Pacific Journal of Mathematics at P.O. Box 969, Carmel Valley, CA 93924 (ISSN 0030-8730) is published monthly except for July and August. Second-class postage paid at Carmel Valley, California 93924, and additional mailing offices. Postmaster: send address changes to Pacific Journal of Mathematics, P.O. Box 969, Carmel Valley, CA 93924.

PUBLISHED BY PACIFIC JOURNAL OF MATHEMATICS, A NON-PROFIT CORPORATION Copyright (C) 1991 by Pacific Journal of Mathematics 